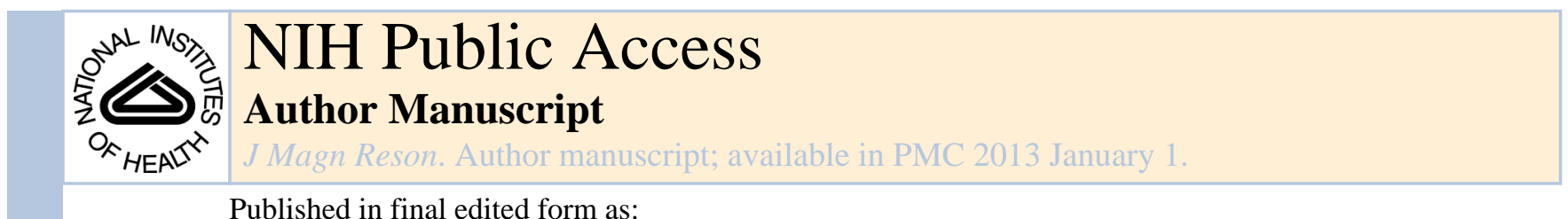

Published in final edited form as:

J Magn Reson. 2012 January ; 214(1): 273-280. doi:10.1016/j.jmr.2011.11.016.

\title{
Measuring $T_{2}$ and $T_{1}$, and Imaging $T_{2}$ without Spin Echoes
}

\author{
G. Wang ${ }^{1,2,{ }^{*}}$, A. M. El-Sharkawy ${ }^{1,{ }^{*}}$, W. A. Edelstein ${ }^{1}$, M. Schär ${ }^{1,3}$, and P. A. Bottomley ${ }^{1,2}$ \\ ${ }_{1}^{1}$ Department of Radiology and Radiological Science, Johns Hopkins University, Baltimore MD \\ ${ }^{2}$ Department of Electrical and Computer Engineering, Johns Hopkins University, Baltimore MD \\ ${ }^{3}$ Philips Healthcare, Cleveland OH, USA
}

\begin{abstract}
During adiabatic excitation, the nuclear magnetization in the transverse plane is subject to $T_{2}$ (spin-spin) relaxation, depending on the pulse length $\tau$. Here, this property is exploited in a method of measuring $T_{2}$ using the ratio of NMR signals acquired with short and long-duration selfrefocusing adiabatic pulses, without spin-echoes. This Dual- $\tau$ method is implemented with $B_{1}$ insensitive rotation (BIR-4) pulses. It is validated theoretically with Bloch Equation simulations independent of flip-angle, and experimentally in phantoms. Dual- $\tau \mathrm{T}_{2}$ measurements are most accurate at short $\mathrm{T}_{2}$ where results agree with standard spin-echo measures to within $10 \%$ for $\mathrm{T}_{2} \leq$ $100 \mathrm{~ms}$. Dual- $\tau$ MRI performed with a long $0^{\circ}$ BIR-4 pre-pulse provides quantitative $T_{2}$ imaging of phantoms and the human foot while preserving desired contrast and functional properties of the rest of the MRI sequence. A single $0^{\circ} \mathrm{BIR}-4$ pre-pulse can provide $\mathrm{T}_{2}$ contrast-weighted MRI and serve as a " $\mathrm{T}_{2}$-prep" sequence with a lower $\mathrm{B}_{1}$ requirement than prior approaches. Finally, a Tri- $\tau$ experiment is introduced in which both $\tau$ and flip-angle are varied, enabling measurement of $\mathrm{T}_{2}$, $\mathrm{T}_{1}$ and signal intensity in just three acquisitions if flip-angles are well-characterized. These new methods can potentially save time and simplify relaxation measurements and/or contrast-weighted NMR and MRI.
\end{abstract}

\section{Keywords}

spin-spin relaxation; measurement; adiabatic pulses; spin-lattice relaxation; MRI; $\mathrm{T}_{1} ; \mathrm{T}_{2}$

\section{INTRODUCTION}

The term adiabatic as applied to NMR excitation pulses refers to frequency and/or amplitude modulated pulses whose effective $\mathrm{B}_{1}$-field in the frame-of-reference rotating at the Larmor frequency change sufficiently slowly such that the nuclear magnetization $\mathbf{M}$ is able to follow it without inducing transitions[1,2]. Adiabatic pulses are highly-valued for their insensitivity to RF and static magnetic field $\left(\mathrm{B}_{0}\right)$ inhomogeneity over ranges determined by the pulse duration $\tau$, flip-angle $\theta$, and transverse RF field $\left(\boldsymbol{B}_{1}\right)$ amplitude and

\footnotetext{
(C) 2011 Elsevier Inc. All rights reserved.

Correspondence: Paul A. Bottomley bottoml@mri.jhu.edu, Division of MR Research, Department of Radiology, Johns Hopkins School of Medicine, 600 N. Wolf Street, Park 311, Baltimore, MD 21287, 410.955.0366.

These authors contributed equally to the work.

Publisher's Disclaimer: This is a PDF file of an unedited manuscript that has been accepted for publication. As a service to our customers we are providing this early version of the manuscript. The manuscript will undergo copyediting, typesetting, and review of the resulting proof before it is published in its final citable form. Please note that during the production process errors may be discovered which could affect the content, and all legal disclaimers that apply to the journal pertain.
} 
frequency sweep [3]. The duration of the pulses is supposed to be shorter than any relaxation processes-whence the term, fast passage.

In the classic experiment, the frequency was swept linearly through resonance [1,2]. Nowadays, adiabatic full-passage (AFP; $\theta=180^{\circ}$ ) and half passage (AHP; $\theta=90^{\circ}$ ) pulses with $\boldsymbol{B}_{\boldsymbol{I}}(\mathrm{t})$ amplitude/frequency-sweeps that vary as $\sin / \cos$, tan/tanh and sech/tanh, offer far superior $\mathrm{B}_{1}$-performance [2-5]. The BIR-4 ( $\mathrm{B}_{1}$-insensitive rotation) pulse, which combines four AHP segments, has further extended adiabaticity to flip-angles that can be arbitrarily set anywhere in the range $|\theta| \leq 180^{\circ}$ [6]. The BIR-4 flip-angle is set by means of two phasejumps between the segments, which can be phase-cycled to improve accuracy [7].

All of these adiabatic pulses, and especially the BIR-4 pulses, are intrinsically longer than conventional hard pulses. To the extent that the magnetization $\mathbf{M}$ evolves in the transverse plane during the pulse, it is subject to transverse $T_{2}$ (spin-spin) decay, even when the pulses are self-refocusing $[3,8,9]$. This dependence is potentially exploitable for measuring $T_{2}$ or for enhancing $T_{2}$ contrast. To date, except for the use of spectral linewidths, $T_{2}$ has been measured with NMR spin-echoes (SEs). The most accurate $T_{2}$ measurements are derived from the Carr-Purcell-Meiboom-Gill (CPMG) technique [10]. SEs are routinely used to provide critically important $\mathrm{T}_{2}$-dependent contrast and $\mathrm{T}_{2}$ measurements in clinical diagnostic magnetic resonance imaging (MRI)[11].

Here we present a new approach for measuring $T_{2}$ in NMR and MRI, and for providing $T_{2}$ contrast in MRI, that does not use SEs. Instead, the $\mathrm{T}_{2}$ dependence of adiabatic pulses is harnessed to measure $T_{2}$ by repeating the NMR or MRI acquisition sequence using one or more different adiabatic pulse lengths $\tau$. We introduce the Dual- $\tau$ method, which provides a $\mathrm{T}_{2}$ measurement from the ratio of NMR signals acquired with short- and long-duration adiabatic pulses. This is analogous to the dual-angle method for measuring the $\mathrm{T}_{1}$ (spinlattice) relaxation time from the ratio of signals acquired with two BIR-4 pulse flip-angles. Indeed, addition of a third acquisition permits the measurement of both $\mathrm{T}_{2}$ and $\mathrm{T}_{1}$ using a combination of different pulse lengths and flip-angles in the Tri- $\tau$ method, also introduced herein. Both the Dual- $\tau$ and the Tri- $\tau$ methods are implemented with self-refocusing BIR-4 pulses and validated by Bloch equation simulations, and by experimental studies of phantoms whose $\mathrm{T}_{2} \mathrm{~S}$ and $\mathrm{T}_{1} \mathrm{~s}$ are measured by standard CPMG, SE and partial saturation (PS) methods. The simulations provide a look-up table or curve which is used to convert measured signal ratios into $T_{2}$ values. $T_{1}$ is determined from a formula analogous to that used for the dual-angle method [12].

Because adiabatic pulses are generally unsuitable for spatially-selective excitation in MRI, implementation of the Dual- $\tau$ method in imaging is most easily accomplished by addition of a $0^{\circ}$ BIR- 4 pre-pulse to the conventional MRI sequence. Since a $0^{\circ}$ pulse does not otherwise affect the nuclear spin dynamics, other desirable MRI contrast and functional properties built into the sequence that follows the pre-pulse can be preserved. A single $0^{\circ}$ BIR- 4 prepulse of length $\tau$ can provide $T_{2}$-contrast or $T_{2}$-weighted MRI, as well as " $T_{2}$-prep" $\left(T_{2^{-}}\right.$ preparation) MRI with a lower $\mathrm{B}_{1}$ threshold than $\mathrm{T}_{2}$-prep sequences employing AHP and AFP pulses [13]. Quantitative Dual- $\tau T_{2}$ imaging can be performed by applying MRI sequences with and without the $0^{\circ} \tau$ pre-pulse. The Dual- $\tau \mathrm{T}_{2}$ MRI method is validated with studies of phantoms and the human foot by comparison with standard CPMG methods.

\section{THEORY}

\section{Dual-т $\mathbf{T}_{2}$ measurements}

The longitudinal and transverse magnetization with magnitudes denoted $M_{z}$ and $M_{x y}$, are affected by both $\mathrm{T}_{1}$ and $\mathrm{T}_{2}$ relaxation following a long adiabatic pulse [9]. At the end of an 
adiabatic pulse with flip angle $\theta$ (time $\left.0^{+}\right), M_{z}\left(0^{+}\right)=\cos \theta M_{z}\left(0^{-}\right) E_{p}^{z}$ and

$M_{x y}\left(0^{+}\right)=\sin \theta M_{x y}\left(0^{-}\right) E_{p}^{x y}$, as compared to the start of the pulse (time $\left.0^{-}\right)$. Here $E_{p}^{z}$ and $E_{p}^{x y}$ are longitudinal and transverse attenuation factors, which are functions of $\tau, \mathrm{T}_{2}, \mathrm{~B}_{1}$ and the maximum frequency sweep, $f_{\max }$, of the pulse, but not $\mathrm{T}_{1}$, provided that $\tau \ll \mathrm{T}_{1}$. After selfrefocusing at the end of the adiabatic pulse, the $\mathrm{T}_{2}$ decay can be written as $E_{p}^{x y}=e^{-g \tau / T_{2}}$, where $g$ is a parameter reflecting the fraction of time spent by the magnetization in the transverse plane during the pulse.

If the residual transverse magnetization prior to time $0^{-}$is crushed [14], the steady-state magnetization after a sequence of adiabatic pulses applied at a repetition period TR comparable to $\mathrm{T}_{1}$ is [9]:

$$
M_{z}\left(0^{+}\right)=M_{0} \frac{\left(1-E_{1}\right) \cos \theta E_{p}^{z}}{1-\cos \theta E_{p}^{z} E_{1}} ; \quad M_{x y}\left(0^{+}\right)=M_{0} \frac{\left(1-E_{1}\right) \sin \theta E_{p}^{x y}}{1-\cos \theta E_{p}^{z} E_{1}}
$$

where $E_{1}=e^{-T R / T_{1}}$ and $\mathrm{M}_{0}$ is the equilibrium nuclear magnetization. If $\theta=90^{\circ}$, the application of two such sequences with the same TR but two different pulse durations $\tau_{1}$ and $\tau_{2}$, will produce steady-state signals $M_{x y}\left(\tau=\tau_{1}\right)=M_{0}\left(1-E_{1}\right) E_{p 1}^{x y}$ and

$M_{x y}\left(\tau=\tau_{2}\right)=M_{0}\left(1-E_{1}\right) E_{p 2}^{x y}$.

The ratio of these signals is:

$$
R=\frac{M_{x y}\left(\tau=\tau_{1}\right)}{M_{x y}\left(\tau=\tau_{2}\right)}=\frac{M_{0}\left(1-E_{1}\right) E_{p 1}^{x y}}{M_{0}\left(1-E_{1}\right) E_{p 2}^{x y}}=\frac{E_{p 1}^{x y}}{E_{p 2}^{x y}}=\frac{E_{p 1}}{E_{p 2}}
$$

where $E_{p 1}$ and $E_{p 2}$ are the attenuation factors for the two pulses. Here we have set $E_{p}=E_{p}^{z}=E_{p}^{x y}$ based on numerical analyses of the Bloch equations with BIR-4 pulses of duration $\tau<40 \mathrm{~ms}$, that shows that the attenuation of the magnetization, $E_{P}=|\mathrm{M}| / \mathrm{M}_{0}$, is independent of flip-angle at least up to $90^{\circ}$ for $200 \leq \mathrm{T}_{1} \leq 1000 \mathrm{~ms}$ and $5 \leq \mathrm{T}_{2} \leq 200 \mathrm{~ms}$. Eq. (2) shows that $\mathrm{R}$ is just a function of $\mathrm{T}_{2}$ which can be derived numerically from the known $\mathrm{B}_{1}$ and $f_{\max }$.

If $\theta=0^{\circ}$, as in the case of a sequence comprised of a $0^{\circ} \mathrm{BIR}-4$ prepulse followed by a short $90^{\circ}$ readout pulse, $M_{x y}$ is attenuated in a similar fashion by $E_{p}{ }^{z}$. The signal following the read-out pulse if two different-duration $0^{\circ} \mathrm{BIR}-4$ pre-pulses are used is

$M_{x y}\left(\tau=\tau_{1}\right)=M_{0}\left(1-E_{1}\right) E_{p 1}^{z}$ and $M_{x y}\left(\tau=\tau_{2}\right)=M_{0}\left(1-E_{1}\right) E_{p 2}^{z}$. Their ratio is $R=\frac{E_{p 1}^{z}}{E_{p 2}^{z}}=\frac{E_{p 1}}{E_{p 2}}$, the same as in Eq. (2).

\section{Tri-T $T_{2}$ and $T_{1}$ measurements}

When TR is short, the Dual- $\tau$ method is limited to choices of $\theta=90^{\circ}$ (or a $\theta=0^{\circ}$ BIR-4 pulse followed by a $90^{\circ}$ ) to cancel troublesome terms in Eq. (1). Incomplete cancellation can occur due to pulse imperfections, incomplete dephasing of residual transverse magnetization, and/or deviations in $E_{p}$ as TR approaches $\mathrm{T}_{2}$. Achieving perfect $90^{\circ}$ pulses is often problematic for in vivo applications such as MRI where the pulses are slice-selective and the RF fields are seldom uniform across the slice. While increasing TR can alleviate this problem, long TRs generally reduce the signal-to-noise (SNR) ratio per unit time and increase the scan time. These limitations are overcome by adding a third acquisition to 
accommodate both $\theta<90^{\circ}$ pulses and short TRs. Importantly, the additional acquisition can permit a simultaneous determination of both $\mathrm{T}_{1}$ and $\mathrm{T}_{2}$.

We call this the Tri- $\tau$ method, in which: 1) a first signal $S_{1}$ is acquired with a conventional short $\alpha \mathrm{RF}$ excitation pulse without adiabatic excitation; 2) a second signal $\mathrm{S}_{2}$ is excited by a $\beta$ adiabatic pulse of duration $\tau_{2}$; and 3 ) a third signal $S_{3}$ is excited by a $\beta$ adiabatic of length $\tau_{3}=2 \tau_{2}$. The three steady-state signals are:

$$
S_{1}=\frac{\left(1-E_{1}\right) \sin \alpha}{\left(1-E_{1} \cos \alpha\right)} M_{0}, S_{2}=\frac{\left(1-E_{1}\right) E_{p 2} \sin \beta}{\left(1-E_{1} E_{p 2} \cos \beta\right)} M_{0} \text { and } S_{3}=\frac{\left(1-E_{1}\right) E_{p 3} \sin \beta}{\left(1-E_{1} E_{p 3} \cos \beta\right)} M_{0}
$$

Using the same $\mathrm{B}_{1}$ and $f_{\max }$ for both adiabatic pulses yields $E_{p}=e^{-g \tau / T_{2}}, E_{p 3}=E_{p 2}{ }^{2}$, and Eq. 3 simplifies to a quadratic:

$$
a E_{p 2}^{2}+b E_{p 2}+c=0
$$

where $a=S_{1} \sin \beta \cos \beta \sin \alpha\left(S_{3}-S_{2}\right), b=S_{1} S_{2} \sin \alpha \cos \alpha \sin \beta-S_{2} S_{3} \sin ^{2} \alpha \cos \beta$, and $c=S_{2} S_{3}$ $\sin ^{2} \alpha \cos \beta-S_{1} S_{3} \sin \alpha \cos \alpha \sin \beta$.

Choosing the root of Eq. (4) that falls in the interval $(0,1)$ yields the $T_{2}$ attenuation factor

$$
E_{p 2}=\frac{-b \pm \sqrt{b^{2}-4 a c}}{2 a}
$$

Substitution of Eq. (5) into Eq. (3) yields the $\mathrm{T}_{1}$ exponential decay factor:

$$
E_{1}=\frac{S_{2} \sin \alpha-S_{1} \sin \beta E_{p 2}}{\left(S_{2} \sin \alpha \cos \beta-S_{1} \cos \alpha \sin \beta\right) E_{p 2}} \text { or } E_{1}=\frac{S_{3} \sin \alpha-S_{1} \sin \beta E_{p 3}}{\left(S_{3} \sin \alpha \cos \beta-S_{1} \cos \alpha \sin \beta\right) E_{p 3}} .
$$

This is basically the dual-angle equation [12] with an $E_{p 2}$-attenuation correction. Thus, from $E_{p 2}$ and $E_{1}$ :

$$
T_{2}=-\frac{g \cdot \tau_{2}}{\ln \left(E_{p 2}\right)} \quad \text { and } \quad T_{1}=-\frac{T R}{\ln \left(E_{1}\right)}
$$

\section{MATERIAL AND METHODS}

\subsection{Numerical simulations}

Simulations of the Bloch equations were performed using Matlab (Mathworks, Natick, MA) [15]. The evolution of magnetization was simulated for proton $\left({ }^{1} \mathrm{H}\right)$ relaxation at $3 \mathrm{~T}$ over the range $20 \leq \mathrm{T}_{2} \leq 200 \mathrm{~ms}$ and $0.1 \mathrm{~s} \leq \mathrm{T}_{1} \leq 1 \mathrm{~s}$ for BIR-4 pulses with $\mathrm{B}_{1}=20 \mu \mathrm{T}, f_{\max }=15 \mathrm{kHz}$, and offset frequencies of $\pm 300 \mathrm{~Hz}$, as used in experiments. Pulses were defined at $5 \mu \mathrm{s}$ intervals with lengths varying from $1 \leq \tau \leq 40 \mathrm{~ms}$, a practical range given limitations in RF pulse power and power deposition. Adiabatic pulse flip-angles were varied from $0 \leq \theta \leq$ $90^{\circ}$. The simulations were used to derive the attenuation factors, $E_{p}^{z}$ and $E_{p}^{x y}$ after long BIR-4 pulses, decay parameter $g$, and the corresponding signal ratio $R$, as a function of $\mathrm{T}_{2}, \mathrm{~T}_{1}, \tau$, and $\theta$ in the Dual- $\tau$ experiment. 
Monte Carlo simulations were performed to evaluate the sensitivity of Dual- $\tau$ and Tri- $\tau$ measurements, to noise. The standard deviation (SD) of the noise was set at $2 \%$ of the signal strength $\left(\mathrm{SNR}=50\right.$ ) elicited by each pulse. The signals, their ratio $R$ for the Dual- $\tau \mathrm{T}_{2}$ experiment, and the solutions to Eq. 7 for the Tri- $\tau$ experiment, were determined for 1000 simulations as a function of $\mathrm{T}_{2}$ up to $80 \mathrm{~ms}$ in the Dual- $\tau$ and Tri- $\tau$ experiments, and $0.1 \mathrm{~s} \leq$ $\mathrm{T}_{1} \leq 1 \mathrm{~s}$ for the Tri- $\tau$ experiment. Note that $\mathrm{T}_{2}$ measured by the Dual- $\tau$ experiment is unaffected by TR for perfect $\theta=90^{\circ}$ pulses.

The utility of a single $0^{\circ} \mathrm{BIR}-4$ pre-pulse for providing $\mathrm{T}_{2}$-contrast, $\mathrm{T}_{2}$-weighting or $\mathrm{T}_{2}$-prep MRI was simulated for BIR-4 pulses with $\tau \leq 45 \mathrm{~ms}, \mathrm{~B}_{1} \leq 100 \mu \mathrm{T}$ and offset frequencies $\leq$ $800 \mathrm{~Hz}$. Results were compared with the AHP/AFP $\mathrm{T}_{2}$-prep sequence of Ref. [13].

\subsection{Phantom preparation}

Twelve phantoms with tissue-comparable relaxation times were prepared with agarose (Type 1-A CAS 9012-36-3: Sigma Chemical, St. Louis, MO, USA) and $\mathrm{CuSO}_{4}$ (CAS 7758-99-8: Acros organics, Geel, Belgium) in de-ionized distilled water. Both agarose and $\mathrm{CuSO}_{4}$ shorten the $\mathrm{T}_{1}$ and $\mathrm{T}_{2}$ of pure water. However, agarose decreases $\mathrm{T}_{2}$ more effectively than $\mathrm{CuSO}_{4}$, which more strongly affects $\mathrm{T}_{1}$. The concentrations of $\mathrm{CuSO}_{4}$ and agarose were adjusted from 0.2 to $1.6 \mathrm{~g} / \mathrm{l}$ and 10 to $110 \mathrm{~g} / \mathrm{l}$ respectively, to provide phantoms with $10 \mathrm{~ms}<\mathrm{T}_{2}<130 \mathrm{~ms}$, and $0.15 \mathrm{~s}<\mathrm{T}_{1}<1.0 \mathrm{~s}[16]$. The ingredients were mixed in a beaker and heated in a microwave oven to dissolve the agarose without introducing too many bubbles. The hot solutions were then sealed in $25 \mathrm{~mm}$ inner diameter (ID) plastic tubes and allowed to cool and gel. Tubes were filled to about 9 or $4 \mathrm{~cm}$ for the Dual- $\tau$ and Tri- $\tau$ experiments respectively.

\subsection{NMR measurements}

All NMR and MRI measurements were done on a 3T Achieva MRI scanner (Philips Healthcare, Best, The Netherlands) using the standard transmit/receive birdcage head coil (maximum $\mathrm{B}_{1}=20 \mu \mathrm{T}$ ). The gel phantoms were set in a plastic foam panel in the center of the coil with long axes parallel to the z-axis. To facilitate $T_{1}$ and $T_{2}$ measurements, NMR data were acquired with one-dimensional chemical shift imaging (1D CSI, $5 \mathrm{~mm}$ resolution; acquisitions per frame, NEX=1) from five aligned phantoms at a time. In all measurements involving BIR-4 pulses, the flip angles were calibrated at the pulse lengths being tested by determining the phase-jump offset at which a $0^{\circ}$ BIR-4 pulse produced near-zero signal $(<2 \%)$ [7], and then verifying that a $90^{\circ}$ BIR-4 pulse with the same offset yielded maximum fully-relaxed signal. Dual- $\tau \mathrm{T}_{2}$ was measured with two $90^{\circ} \mathrm{BIR}-4$ pulses of lengths $\tau_{1}=5 \mathrm{~ms}$ $\left(f_{\max }=12 \mathrm{kHz}\right)$ and $\tau_{2}=35 \mathrm{~ms}\left(f_{\max }=15 \mathrm{kHz}\right)$, the shortest TE $(1.8 \mathrm{~ms})$ and TR $=4 \mathrm{~s}$.

Reference $T_{2}$ and $T_{1}$ relaxation times were measured at $3 T$ using standard SE and PS NMR methods, respectively. Individual SE data was obtained using 1D CSI with 8 different echo times $(\mathrm{TE}=14,30,50,75,100,150,200,300 \mathrm{~ms})$. TR was set to 4.0 s to allow for complete signal recovery. $\mathrm{T}_{1}$ was measured using PS sequences with $\mathrm{TR}=0.25,0.5,1$, and $3 \mathrm{~s}$.

Because relaxation times can change slowly over time, these measurements were repeated whenever a dual- or Tri- $\tau$ study was done. The effect of offset frequency on $T_{2}$ accuracy was tested separately in CSI experiments on five of the short phantoms with $\mathrm{T}_{2} \mathrm{~s}$ of 30-60ms at offset frequencies of $\leq 300 \mathrm{~Hz}$.

The CSI experiments yielded approximately 5 slices in the CSI direction from each phantom. The free induction decays (FID) acquired from the three middle slices in each phantom were fitted using jMRUI software (available from www.mrui.uab.es)[17] to determine the peak areas. These values were used to determine the signal ratio at the two $\tau$ values. Dual- $\tau \mathrm{T}_{2}$ was then determined from a look-up curve generated by the numerical 
analysis (see Results). Reference $\mathrm{T}_{1}$ and $\mathrm{T}_{2}$ measurements obtained by conventional $\mathrm{SE}$ and PS methods were determined from the same three slices by fitting the data sets to $\left\{w \exp \left(-T E / T_{2}\right)\right\}$ and $\left\{u-v \exp \left(-T R / T_{1}\right)\right\}$ with $u, v$ and $w$ constants. Mean reference relaxation times from the phantoms used in the Dual- $\tau$ studies are listed in Table 1.

The Tri- $\tau$ method was also validated in 1DCSI studies of $12 \times 1.3 \mathrm{~cm}$ ID and $4 \times 2.5 \mathrm{~cm}$ ID phantoms with $169 \leq \mathrm{T}_{1} \leq 890 \mathrm{~ms}$ and $31 \leq \mathrm{T}_{2} \leq 129 \mathrm{~ms}$. We used $\tau_{3}=2 \tau_{2}=20 \mathrm{~ms}$, and $\mathrm{TR}=300 \mathrm{~ms}$ for all experiments. Shorter TRs were limited by RF power restrictions. $\mathrm{S}_{1}$ was acquired with a (non-adiabatic) $75 \mu$ s hard pulse nominally set to $\alpha=15^{\circ}$. The flip-angle was $14^{\circ}$ for the short phantoms as determined from $B_{1}$ field profiles measured separately, and $13.5^{\circ}$ for the long phantoms. The $\beta$-pulse used to acquire $S_{2}$ and $S_{3}$ was a $60^{\circ}$ BIR-4 pulse. The choice of the nominal $\alpha=15^{\circ}$ and $\beta=60^{\circ}$ pair for the Tri- $\tau$ experiments was based on the dual-angle $\mathrm{T}_{1}$ method [12].

\subsection{MRI measurements}

The BIR-4 pulse is unsuited to spatial localization. Thus for MRI applications, either multidimensional phase-encoding must be used (with generally unpalatable scan times), or the BIR-4 pulse must be applied in conjunction with an additional spatial localization pulse. We adopt the latter approach by adding a $0^{\circ}$ BIR-4 pulse before the slice-selective pulse in a standard gradient-echo (FFE) MRI pulse sequence, as simulated.

Dual- $\tau$ MRI was performed on the phantom set with a two-dimensional (2D) FFE MRI sequence and $0^{\circ} \mathrm{BIR}-4$ pulses with the same $\tau, f_{\max }$ and $\mathrm{B}_{1}$ used in the NMR experiments (acquisition matrix, $152 \times 154$; field-of-view, FOV=97 $\times 10 \times 230 \mathrm{~mm}^{3}$; slice thickness $=10 \mathrm{~mm}$; TR=2s; minimum TE=2.5ms). The delay between the BIR-4 and $(1 \mathrm{~ms})$ slice-selective pulses was $1.5 \mathrm{~ms}$. Because the excitation pulse and inter-pulse delay are unchanged in this Dual- $\tau$ MRI experiment, the same curve from the numerical analysis was used to obtain $T_{2}$. The sequence timing diagram is shown in Fig 1. Reference $T_{2}$ values from conventional MRI were obtained from the phantoms using a three-dimensional (3D) CPMG sequence $(32$ echoes, TE step $=7.21 \mathrm{~ms}, \mathrm{TR}=461 \mathrm{~ms}, 2.5 \mathrm{~ms}$ excitation pulse $1.5 \mathrm{~ms}$ after the BIR-4 pulse). $T_{2}$ was measured in images by determining the ratio $R$ on a pixel-by-pixel basis for the Dual- $\tau$ method, and by fitting the SE pixels to an exponential to obtain reference $T_{2}$ images. Pixel-average $T_{2}$ values within each phantom are reported (means \pm standard deviation, SD). The consistency of $\mathrm{T}_{2}$ measured by SE 1D CSI and CPMG MRI was verified with measurements on five additional phantoms with $\mathrm{T}_{2}=30-60 \mathrm{~ms}$.

Human studies for this project were approved by the Johns Hopkins Institutional Review Board. The foot was chosen based on the expected short $\mathrm{T}_{2} \mathrm{~s}$ of muscle and cartilage [18], for which analysis showed the Dual- $\tau$ method to be well-suited. A healthy volunteer was positioned supine with the foot in the head coil, and Dual- $\tau T_{2}$ MRI performed using two acquisitions of a coronal 3D FFE sequence, one employing a $0^{\circ}$ BIR-4 pre-pulse (matrix size, $112 \times 112$; slice thickness $=6 \mathrm{~mm} ; \mathrm{FOV}=42 \times 100 \times 100 \mathrm{~mm}^{3}$, TR $=2 \mathrm{~s}, \mathrm{TE}=4.1 \mathrm{~ms}$ $=$ minimum). Reference $T_{2}$ MRI of the foot was performed with the 32-echo CPMG sequence (same matrix, slice thickness and FOV as for Dual- $\tau$ FFE; TE step $=5.39 \mathrm{~ms}$; $\mathrm{TR}=625 \mathrm{~ms}) . T_{2}$ images and average $T_{2}$ values were calculated the same way as in the phantom studies. 


\section{RESULTS}

\section{Numerical analysis}

The attenuation factor, $E_{p}$ as a function of $\mathrm{T}_{2}$ is plotted in Fig 2 for four BIR-4 pulses of

duration 5-35ms. The curves all fit accurately to the exponential $E_{p}\left(T_{2}\right)=\exp \left(-0.81 \frac{\tau}{T_{2}}\right)$. In this case, $g=0.81$. A side-effect of this result is that the $\mathrm{T}_{2}$ attenuation from a single long duration $\tau_{2}$ adiabatic pulse is the same as that resulting from concatenation of $n$ short $\tau_{1}$ pulses of the same total duration $\tau_{2}$, except for an approximately $n$-fold increase in $\mathrm{B}_{1}$ to achieve adiabaticity. The result in Fig. 2 is also independent of flip-angle for $0^{\circ} \leq \theta \leq 90^{\circ}$ and for long TR, reflecting the fact that the BIR-4 flip-angle is set by two opposite phasejumps of duration much less than $\tau, T_{2}$ and $T_{1}$. In the adiabatic region wherein $\theta$ is independent of $\mathrm{B}_{1}, g$ is relatively insensitive to $f_{\max }$, for example, varying from 0.79 to 0.81 for $12 \mathrm{kHz} \leq f_{\max } \leq 15 \mathrm{kHz}$ and $\mathrm{B}_{1}=20 \mu \mathrm{T}$.

The sensitivity and accuracy of the Dual- $\tau T_{2}$ experiment are improved by selecting BIR- 4 pulses with very different pulse lengths, notwithstanding RF power constraints. Pulses shorter than $5 \mathrm{~ms}$ have little attenuation but are limited by peak pulse power. Long pulses are limited by the RF power amplifier's ability to sustain the pulse, as well as by the spectral bandwidth of the sample. The ratio of the signals from the $\tau_{1}=5 \mathrm{~ms}$ and $\tau_{2}=35 \mathrm{~ms}$ pulses is plotted in Fig 3(a). The curve is fit by $R\left(T_{2}\right)=\exp \left(-24.3 / T_{2}\right)$ independent of $\theta$. Sensitivity is maximum over the steepest (short- $\mathrm{T}_{2}$ ) region of the curve, with the $\tau_{1} / \tau_{2}=5 / 35 \mathrm{~ms}$ pair providing reasonable $\mathrm{T}_{2}$ resolution up to 70 or $80 \mathrm{~ms}$. Thus, $\mathrm{T}_{2}$ can be read from Fig. 3(a) using the measured signal ratio. The variation in $\mathrm{T}_{2}$ determined from the ratio curve is plotted as a function of $\mathrm{T}_{1}$ in Fig. 3(b). The result varies by $<1 \%$ for $0.6 \mathrm{~s} \leq \mathrm{T}_{1} \leq 1.0 \mathrm{~s}$, and $<6 \%$ for $0.2 \mathrm{~s} \leq \mathrm{T}_{1} \leq 1.0 \mathrm{~s}$, but begins to introduce errors as $\mathrm{T}_{2}$ approaches $\mathrm{T}_{1}$. The results of the analysis of the affect of offset frequencies of up to $\pm 300 \mathrm{~Hz}$, show that $\mathrm{T}_{2}$ varies by $<6.5 \%$ for $30 \mathrm{~ms} \leq \mathrm{T}_{2} \leq 130 \mathrm{~ms}$, $\mathrm{TR}=\mathrm{T}_{1}=1 \mathrm{~s}$ and $\mathrm{B}_{1}=20 \mu \mathrm{T}$.

The results of the Monte Carlo simulations are exemplified in Fig. 4. For the Dual- $\tau \mathrm{T}_{2}$ experiment performed with $\tau_{1} / \tau_{2}=5 / 35 \mathrm{~ms}$ pulses and signals with $2 \% \mathrm{SD}$, the errors are essentially independent of TR for $0.1 \mathrm{~s} \leq \mathrm{TR} \leq 1.0 \mathrm{~s}$. Over this range, the mean error in $\mathrm{T}_{2}$ is less than $6 \% \pm 9 \%$ (SD) of $\mathrm{T}_{2}$ for $\mathrm{T}_{2} \leq 80 \mathrm{~ms}$ and $0.3 \mathrm{~s} \leq \mathrm{T}_{1} \leq 1 \mathrm{~s}$ (Fig. $4 \mathrm{a}$ ). On the other hand, the simulated Tri- $\tau$ experiment with $\tau_{3}=2 \tau_{2}=20 \mathrm{~ms}$ is less accurate on average, with a mean error varying from $-13 \%$ to $+6 \%$ of $\mathrm{T}_{2}$ (with up to $\pm 30 \% \mathrm{SD}$ scatter) for $\mathrm{T}_{2} \leq 80 \mathrm{~ms}$ over the ranges $0.1 \mathrm{~s} \leq \mathrm{TR} \leq 1.0 \mathrm{~s}$ and $0.1 \leq \mathrm{T}_{1} \leq 1 \mathrm{~s}$ (Fig. $4 \mathrm{~b}$ ). When $\tau_{3}$ is set to $35 \mathrm{~ms}-$ the same as $\tau_{2}$ in the Dual- $\tau$ experiment-the mean error decreases to $<13 \% \pm 17 \%$ (SD) of $\mathrm{T}_{2}$ for the same $\mathrm{T}_{1} \mathrm{~s}$ and TRs (eg, Fig. 4c).

The accuracy of $\mathrm{T}_{1}$ in the Monte Carlo simulations of the Tri- $\tau$ experiment is better than $1 \%$ of $\mathrm{T}_{1}$ with a $9-15 \%(\mathrm{SD})$ scatter, essentially independent of pulse length for $0.3 \leq \mathrm{T}_{1} \leq 1 \mathrm{~s}$ and $30 \leq \mathrm{T}_{2} \leq 130 \mathrm{~ms}$, as shown in Fig. $4(\mathrm{~d})$ for $\mathrm{TR}=0.3 \mathrm{~s}$. At shorter $\mathrm{T}_{1} \mathrm{~s}(<\mathrm{TR})$, the scatter in $\mathrm{T}_{1}$ increases as the long TR becomes sub-optimal for measuring $\mathrm{T}_{1}$ [8]. In this case, reducing TR to $0.1 \mathrm{~s}$, reduces the scatter back to $9-15 \%$ of $\mathrm{T}_{1}$ for $0.1 \leq \mathrm{T}_{1} \leq 1.0 \mathrm{~s}$.

The performance of a $45 \mathrm{~ms} 0^{\circ} \mathrm{BIR}-4$ pre-pulse for $\mathrm{T}_{2}$-prep MRI is shown in Fig. 5 for a sample with $\mathrm{T}_{2}=55 \mathrm{~ms}$. The $\mathrm{B}_{1}$ threshold is approximately $5 \mu \mathrm{T}$, and the pulse attenuates $M_{z}$ by $\exp \left(-0.81 \times 45 / \mathrm{T}_{2}\right) \approx 0.5$ over a broad range of $\mathrm{B}_{1}$ and offset frequencies.

\section{Experiments}

Results from the ${ }^{1} \mathrm{H}$ 1D CSI Dual- $\tau$ validation experiments on the 10 phantoms are plotted in Fig. 6. The $T_{1}$ of these phantoms was 0.6-0.8s (Table 1). The $T_{2} s$ were determined from 
Fig. 3. Below $\mathrm{T}_{2}=70 \mathrm{~ms}$, Dual- $\tau \mathrm{T}_{2}$ values differ from $\mathrm{SE}$ values by $\leq 3 \%$. The accuracy of $\mathrm{T}_{2}$ varied $\leq 7 \%$ for offset frequencies $\leq 300 \mathrm{~Hz}$ compared to $\mathrm{T}_{2}$ measured on resonance, consistent with the simulations.

Dual- $\tau T_{2}$-weighted and $T_{2}$-image results from the phantoms are shown in Fig 7 . The phantoms are labeled with the corresponding average $T_{2}$ values measured by standard 32echo CPMG MRI (Fig. 7a), and by Dual- $\tau$ MRI (Fig. 7b). The $T_{2}$ values agree with the CPMG results within $5 \%$ up to $70 \mathrm{~ms}$, and $10 \%$ up to $100 \mathrm{~ms}$. The consistency check on $\mathrm{T}_{2}$ measured by CPMG MRI vs SE ${ }^{1} \mathrm{H}$ 1D CSI showed no significant differences (CPMG vs. SE results: $31.1 \pm 0.3$ vs $31.5 \pm 0.3 \mathrm{~ms}, 38.9 \pm 0.5$ vs $38.3 \pm 1.8 \mathrm{~ms}, 36.7 \pm 0.5$ vs $37.5 \pm 0.3 \mathrm{~ms}$, $60 \pm 6.1$ vs $59.4 \pm 2.3 \mathrm{~ms}, 58.6 \pm 5$ vs $57.7 \pm 0.7 \mathrm{~ms})$.

Dual- $\tau$ and standard SE $T_{2}$ MRI of the foot are compared in Fig 8. Muscle $T_{2}$ values from the Dual- $\tau$ image are $29.4 \pm 1.2 \mathrm{~ms}, 28.2 \pm 1.6 \mathrm{~ms}$, and $30.6 \pm 1.3 \mathrm{~ms}$; as compared to $29.8 \pm 2.8 \mathrm{~ms}, 29.8 \pm 4.7 \mathrm{~ms}$, and $28.8 \pm 4.7 \mathrm{~ms}$ in the same annotated volumes in the CPMG image. These agree with published values for muscle of $32 \pm 2 \mathrm{~ms}$ at 3T.[18]. In marrow, $\mathrm{CPMG} \mathrm{T}_{2}$ was $106 \pm 4.3 \mathrm{~ms}$ as compared to Dual $-\tau \mathrm{T}_{2}=135 \pm 13 \mathrm{~ms}$ in the same volume, and a prior value of $133 \pm 6 \mathrm{~ms}$ for marrow[18].

$T_{2}$ and $T_{1}$ values measured from the Tri- $\tau$ experiments on phantoms are compared with SE and PS $\mathrm{T}_{1}$ and $\mathrm{T}_{2}$ values in Fig. 9. The values show good agreement for all phantoms.

\section{DISCUSSION}

Long adiabatic pulses such as BIR-4 are self-refocusing but are subject to $\mathrm{T}_{2}$ decay, resulting in attenuation by the end of the pulse. We have shown for the first time that $T_{2}$ measurements and $\mathrm{T}_{2}$ image contrast can be obtained using these adiabatic pulse properties as distinct from conventional methods that use spin-echoes or $180^{\circ}$ refocusing pulses. In particular, we have presented new NMR and MRI pulse sequences for measuring and imaging $\mathrm{T}_{2}$ that can be performed in just two acquisitions employing long and short duration adiabatic pulses for NMR, or a long and no adiabatic pulse for MRI. These Dual- $\tau$ methods were validated for BIR-4 pulses by numerical analysis using the Bloch Equations and experimental ${ }^{1} \mathrm{H}$ NMR and MRI studies of phantoms with different $\mathrm{T}_{2} \mathrm{~s}$ and MRI of the human foot, compared to conventional SE $\mathrm{T}_{2}$ measurements (Figs. 3-7). Moreover, extension of the Dual- $\tau$ to the Tri- $\tau$ method resulted in a technique that not only delivers $\mathrm{T}_{2}$ measurements but $T_{1}$ as well. The Tri- $\tau$ method was also validated by both simulations (Fig. 4) and experimental measurements on phantoms (Fig. 9).

Compared to a two-acquisition SE sequence, the efficiency of the Dual- $\tau$ method for determining $T_{2}$ is the same. Although in principle $T_{2}$ could be determined from a single CPMG sequence in half the time of a Dual- $\tau$ measurement, the acquisition window for the Dual- $\tau$ method is not constrained by the echo spacing and could conceivably benefit from reducing the bandwidth to offset loss in SNR per unit time compared to CPMG. In the Tri- $\tau$ method, the $\mathrm{T}_{1}$-determining portion is based on the Dual-angle $\mathrm{T}_{1}$ method [12], and hence it has the same efficiency except for the SNR loss due to the $T_{2}$ decay that is encoded. The options comparable to the Tri- $\tau$ method for obtaining both $\mathrm{T}_{1}$ and $\mathrm{T}_{2}$ in three acquisitions are limited and seldom reduced-to-practice. Conceivably, one could use an SE or CPMG sequence to obtain $\mathrm{T}_{2}$ and a PS [19], SSFP [20] or a Dual-angle [12] sequence to obtain $\mathrm{T}_{1}$, in which case the total number of acquisitions would be three or more. The Tri- $\tau$ method would be comparable or faster than these options.

As with existing techniques for measuring relaxation times, the Dual- $\tau$ and Tri- $\tau$ methods can be adversely affected by $\mathrm{B}_{1}$-field nonuniformity when the adiabatic pulses are combined with conventional pulses such as those provided by MRI sequences (Fig. 1). The combined 
effect of an imperfect slice profile and $B_{1}$ inhomogeneity-to which higher-field MRI systems are intrinsically more susceptible-is significant. In order to cancel the denominator in Eq. (1) and obtain a ratio $\mathrm{R}$ from which $\mathrm{T}_{2}$ can be determined using the Dual- $\tau$ method, we must have either $\theta=90^{\circ}$ or a long TR. Obtaining an exact $\theta=90^{\circ}$ slice-selective excitation pulse at 3T depends on the accuracy of the scanner's set-up routine. Our Dual- $\tau$ MRI studies of the foot benefited from the use of a 3D (slab-select) pulse sequence for both the Dual- $\tau$ and reference CPMG MRI; selection of just the middle slices; and a longer TR than we would have liked because of errors in the slice-selective $90^{\circ}$ pulse. Thus, relative to other $\mathrm{T}_{1}$ and $\mathrm{T}_{2}$ methods, the sensitivity of the Dual- $\tau$ and Tri- $\tau$ methods to $\mathrm{B}_{1}$ inhomogeneity depends on the pulses being used. Dual- $\tau$ errors in $\mathrm{T}_{2}$ were $\leq 7 \%$ for up to $\pm 300 \mathrm{~Hz}$ frequency offsets, due to the bandwidth of the BIR-4 pulse. However, performing $\mathrm{SE} \mathrm{T}_{2}$ experiments with $35 \mathrm{~ms}$ BIR-4 pulses is unrealistic and therefore not directly comparable. All methods employing adiabatic pulses would be expected to exhibit better immunity to $\mathrm{B}_{1}$ variations than those that do not employ adiabatics.

The delays of several milliseconds added between the $0^{\circ}$ BIR- 4 pulse, the slice-selective pulse and the echo-times for the MRI sequences, will affect the total attenuation factor, reducing the SNR a little. This did not seem to affect $T_{2}$ in the Dual- $\tau$ MRI experiment where the delay is the same in both cases. Although the $S_{1}$ acquisition of the Tri- $\tau$ experiment does not have a delay while the $S_{2}$ and $S_{3}$ acquisitions-do, the Tri- $\tau T_{2}$ accuracy was also apparently unaffected.

The accuracy of $T_{1}$ s measured in the Tri- $\tau$ NMR experiment will depend critically on the accuracy of the remaining non-adiabatic low-angle $\left(15^{\circ}\right)$ NMR excitation pulses set by the scanner [12]. If MRI is not intended, an adiabatic $15^{\circ}$ pulse could avoid this problem provided its duration is $\ll \tau_{2}$ to avoid a significant $3^{\text {rd }} E_{p 1}$ term in Eq (3). Meanwhile, the accuracy of $\mathrm{T}_{1}$ imaging using a Tri- $\tau$ MRI sequence wherein the $60^{\circ}$ BIR-4 pulses are replaced by $0^{\circ}$ BIR-4 [6] or BIRP [7] pulses, and nominal $60^{\circ}$ slice-selective MRI pulses are used for $S_{2}$ and $S_{3}$ with a $15^{\circ}$ slice-selective MRI pulse for $S_{1}$, depends on the accuracy with which $B_{1}$ and/or the flip-angles are calibrated. Nevertheless, the Tri- $\tau$ pulse sequence is presently unique in demonstrating a potential for measuring and imaging $\mathrm{T}_{1}, \mathrm{~T}_{2}$ and proton density from just 3 (albeit steady-state) acquisitions all of which are FIDs.

That the decay in magnetization during the BIR-4 pulse is essentially independent of the flip-angle, enables decoupling of $\mathrm{T}_{2}$ from the flip-angle, even allowing a $\mathrm{T}_{2}$ attenuation effect with otherwise zero excitation. In this application, the $0^{\circ} \mathrm{BIR}-4$ pulse serves as a $\mathrm{T}_{2}$ filter, removing the short $\mathrm{T}_{2}$ components (Fig. 7a), while preserving the longer ones for an FID generated by a subsequent conventional excitation. This could be useful in spectroscopy for removing unwanted short- $\mathrm{T}_{2}$ components that generate broad baselines. Also, the use of pre-pulses to add $\mathrm{T}_{2}$ contrast bears similarity to $\mathrm{T}_{2}$-prep MRI sequences, especially those employing adiabatic pulses [13]. Adiabatic $\mathrm{T}_{2}$-prep MRI uses several very short $90^{\circ}$ AHP and $180^{\circ}$ AFP pulses with gaps between them to allow $\mathrm{T}_{2}$ relaxation [13]. By using a (i) single, (ii) long, (iii) $0^{\circ}$ BIR-4 or BIRP pulse, (iv) with no gaps, our sequence differs from that $\mathrm{T}_{2}$-prep sequence in four ways. Moreover, the present work extends the application from providing $\mathrm{T}_{2}$ contrast, to providing $\mathrm{T}_{2}$ measurements.

The use of a single long $0^{\circ}$ BIR- 4 pulse for $\mathrm{T}_{2}$-prep instead of the short AHP/AFP pulses [13] may offer some advantage. The long BIR-4 (or BIRP) pulse has a much lower $\mathrm{B}_{1^{-}}$ threshold to achieve adiabaticity than the short AHP, or AFP pulses. As a consequence, it requires much lower peak power. For example, the simulations of a 45ms AHP/AFP T $\mathrm{T}_{2}$-prep sequence from Fig. 4a of Ref. [13] showed an adiabatic threshold requirement for $\mathrm{B}_{1}$ of about $20 \mu \mathrm{T}$. Performing the same simulation with the same $\mathrm{T}_{1}$ and $\mathrm{T}_{2}$ here, showed that a 
single $45 \mathrm{~ms} 0^{\circ}$ BIR-4 pre-pulse had a $\mathrm{B}_{1}$ threshold of $\sim 5 \mu \mathrm{T}$, or $1 / 4$ that of the AHP/AFP $\mathrm{T}_{2}-$ prep sequence (Fig. 5).

Another question is whether the Dual- $\tau$ experiment with long adiabatic pulses measures either the inhomogeneously broadened $T_{2}\left(T_{2} *\right)$ or the $T_{1}$ in the rotating frame $\left(T_{1 \rho}\right)$, as distinct from a pure $\mathrm{T}_{2}$. First, $\mathrm{T}_{2} *$ results from local $\mathrm{B}_{0}$-field inhomogeneity or offset frequency, to which a BIR-4 pulse operating above its adiabatic threshold is insensitive over a range of several hundred $\mathrm{Hz}[12]$. In the present studies, both analysis and experiments showed that $T_{2}$ varies by $\leq 7 \%$ for offset frequencies in the range $\pm 300 \mathrm{~Hz}$. Second, $T_{1 \rho}$ measures $T_{1}$ at the much lower NMR frequency corresponding to the $B_{1}$ field. Although it is not explicitly present in the Bloch Equations, $T_{1 \rho}$ approaches $T_{2}$ as $B_{1}$ goes to zero and behaves like a combination of low-frequency $T_{1}$ and $T_{2}$ [21]. As such, changes in $T_{1}$ might be expected to affect Dual- $\tau T_{2}$ if it were sensitive to $T_{1 \rho}$. However, analysis of the Dual- $\tau$ experiment showed variations $<6 \%$ in $\mathrm{T}_{2}$ over a 5 -fold range of $\mathrm{T}_{1}$ (Fig. 3b). In experiments, Dual- $\tau \mathrm{T}_{2}$ measured at a $\mathrm{B}_{1}$ of $13.5 \mu \mathrm{T}$ (knee coil) did not differ by more than $3 \%$ from those measured with $\mathrm{B}_{1}=20 \mu \mathrm{T}$ (head coil) on the same short- and long- $\mathrm{T}_{2}$ phantoms. Thus, $\mathrm{T}_{2}{ }^{*}$ and $T_{1 \rho}$ do not appear to be significant factors affecting Dual- $\tau T_{2}$ measurements in this work.

In conclusion, the Dual- $\tau$ method provides a new option for measuring $\mathrm{T}_{2}$ without requiring any spin echoes, at least for short $\mathrm{T}_{2}$ tissues such as muscle, cartilage and white matter [22] where the signal ratio affords adequate $\mathrm{T}_{2}$ resolution (Fig $3 \mathrm{a}$ ). The same property delivered with an otherwise neutral $0^{\circ}$ flip-angle self-refocusing adiabatic pulse can provide $T_{2}$ imaging, $T_{2}$-weighting, $T_{2}$-filtering (Figs. 6, 7), or $T_{2}$-prep (Fig. 9). At the expense of one additional acquisition, the Tri- $\tau$ experiment offers the potential for obtaining all of the $T_{2}, T_{1}$ and, because the nuclear density derives directly from the fully-relaxed signal, the proton or signal density information, in just three acquisitions-arguably the minimum possible. The caveat is the requirement for accurate setting and knowledge of the flip-angles. These new methods can potentially save time and simplify relaxation measurements and/or contrastweighted NMR and MRI.

\section{Acknowledgments}

We thank Terri Brawner from the Kennedy Krieger Institute for the loan of a knee coil, and Dr Refaat E. Gabr, of the Division of MR Research, for helpful discussions and assistance with human studies. The work was supported by NIH grant R01 EB007829.

\section{References}

1. Abragam, A. The Principles of Nuclear Magnetism. Oxford: Clarendon Press; 1961. p. 599

2. Norris DG. Adiabatic radiofrequency pulse forms in biomedical nuclear magnetic resonance. Concepts in Magnetic Resonance. 2002; 14:89-101.

3. De Graaf RA, Nicolay K. Adiabatic rf pulses: Applications to in vivo NMR. Concepts in Magnetic Resonance. 1997; 9:247-268.

4. Silver MS, Joseph RI, Hoult DI. Highly selective [pi]/2 and [pi] pulse generation. Journal of Magnetic Resonance. 1984; 59:347-351.

5. Hardy CJ, Edelstein WA, Vatis D. Efficient adiabatic fast passage for NMR population inversion in the presence of radiofrequency field inhomogeneity and frequency offsets. J Magn Reson. 1986; 66:470-482.

6. Garwood M, Ke Y. Symmetric pulses to induce arbitrary flip angles with compensation for rf inhomogeneity and resonance offsets. Journal of Magnetic Resonance. 1991; 94:511-525.

7. Bottomley PA, Ouwerkerk R. BIRP: an improved implementation of low-angle adiabatic (BIR-4) excitation pulses. J Magn Reson Ser A. 1993; 103:242-244. 
8. Robin Bendall M, Garwood M, Uğurbil K, Pegg DT. Adiabatic refocusing pulse which compensates for variable of power and off-resonance effects. Magnetic Resonance in Medicine. 1987; 4:493499. [PubMed: 3600255]

9. El-Sharkawy AM, Schar M, Ouwerkerk R, Weiss RG, Bottomley PA. Quantitative Cardiac ${ }^{31} \mathrm{P}$ Spectroscopy at 3 Tesla Using Adiabatic Paulses. Magnetic Resonance in Medicine. 2009; 61:785795. [PubMed: 19195018]

10. Meiboom S, Gill D. Modified Spin-Echo Method for Measuring Nuclear Relaxation Times. Review of Scientific Instruments. 1958; 29:688-691.

11. Bottomley, PA.; Edelstein, WA. NMR imaging of the transverse relaxation time using multiple spin echo sequences. US Patent. 4,521,733. June 4. 1985

12. Bottomley PA, Ouwerkerk R. The Dual-Angle Method for Fast, Sensitive T1 Measurement in Vivo with Low-Angle Adiabatic Pulses. Journal of Magnetic Resonance, Series B. 1994; 104:159167.

13. Nezafat R, Ouwerkerk R, Derbyshire AJ, Stuber M, McVeigh ER. Spectrally selective B1insensitive T2 magnetization preparation sequence. Magnetic Resonance in Medicine. 2009; 61:1326-1335. [PubMed: 19319903]

14. Bottomley, PA.; Edelstein, WA. Method of eliminating effects of spurious free induction decay NMR signal caused by imperfect 180 degrees pulses. US Patent. 4,484,138. Nov 20. 1984

15. Bittoun J, Taquin J, Sauzade M. A computer algorithm for the simulation of any Nuclear Magnetic Resonance (NMR) imaging method. Magnetic Resonance Imaging. 1984; 2:113-120. [PubMed: 6530920]

16. Yoshimura K, Kato H, Kuroda M, Yoshida A, Hanamoto K, Tanaka A, Tsunoda M, Kanazawa S, Shibuya K, Kawasaki S, Hiraki Y. Development of a tissue-equivalent MRI phantom using carrageenan gel. Magnetic Resonance in Medicine. 2003; 50:1011-1017. [PubMed: 14587012]

17. Naressi A, Couturier C, Devos JM, Janssen M, Mangeat C, Beer Rd, Graveron-Demilly D. Javabased Graphical User Interface for the MRUI Quantitation Package. MAGMA. 2001; 12:141-152. [PubMed: 11390270]

18. Gold GE, Han E, Stainsby J, Wright G, Brittain J, Beaulieu C. Musculoskeletal MRI at 3.0 T: Relaxation Times and Image Contrast. Am J Roentgenol. 2004; 183:343-351. [PubMed: 15269023]

19. Liu X, Feng Y, Lu ZR, Morrell G, Jeong EK. Rapid simultaneous acquisition of T1 and T2 mapping images using multishot double spin-echo EPI and automated variations of TR and TE (ms-DSEPI-T12). NMR in Biomedicine. 2010; 23:97-104. [PubMed: 19777499]

20. Deoni SCL, Rutt BK, Peters TM. Rapid combined T1 and T2 mapping using gradient recalled acquisition in the steady state. Magnetic Resonance in Medicine. 2003; 49:515-526. [PubMed: 12594755]

21. Moran PR, Hamilton CA. Near-resonance spin-lock contrast. Magnetic Resonance Imaging. 1995; 13:837-846. [PubMed: 8544655]

22. Gelman N, Gorell JM, Barker PB, Savage RM, Spickler EM, Windham JP, Knight RA. MR Imaging of Human Brain at 3.0 T: Preliminary Report on Transverse Relaxation Rates and Relation to Estimated Iron Content. Radiology. 1999; 210:759-767. [PubMed: 10207479] 


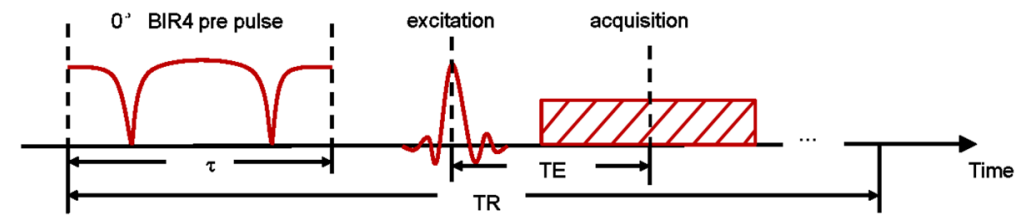

Fig 1.

Timing diagram of a sequence employing one of $0^{\circ}$ BIR- 4 pre-pulses for the Dual- $\tau$ MRI method. In MRI experiments TE was set to a minimum (4.8ms), TR at $2 \mathrm{~s}$, and the gap between the pre- and excitation-pulses was $\sim 1.5 \mathrm{~ms}$. 


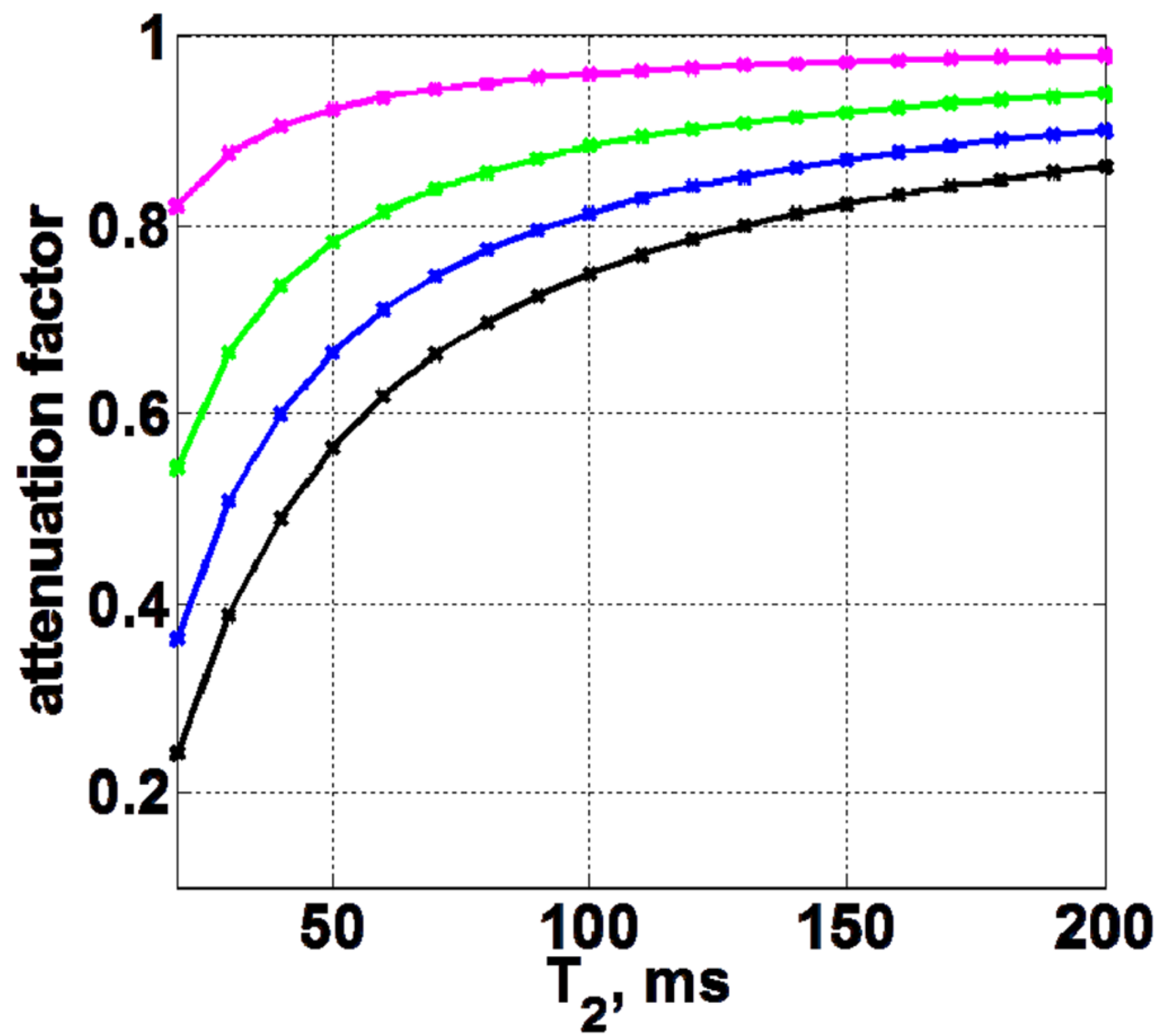

Fig 2.

Numerical simulation of the attenuation factor $E_{P}=|\mathrm{M}| / \mathrm{M}_{0}$ of the magnetization after a BIR-4 pulse of duration 5ms (magenta), $15 \mathrm{~ms}$ (green), 25ms (blue) and $35 \mathrm{~ms}$ (black), as a function of $\mathrm{T}_{2}$ from 20 to $200 \mathrm{~ms}\left(\mathrm{~T}_{1}=1 \mathrm{~s}, \mathrm{TR}=\infty\right.$ for complete longitudinal relaxation) with $f_{\max }=15 \mathrm{kHz}$ and $\mathrm{B}_{1}=20 \mu \mathrm{T}$. The curves are exponential fits to the numericallydetermined points (stars) from which $g=0.81$ and valid for $\theta$ up to $90^{\circ}$. 

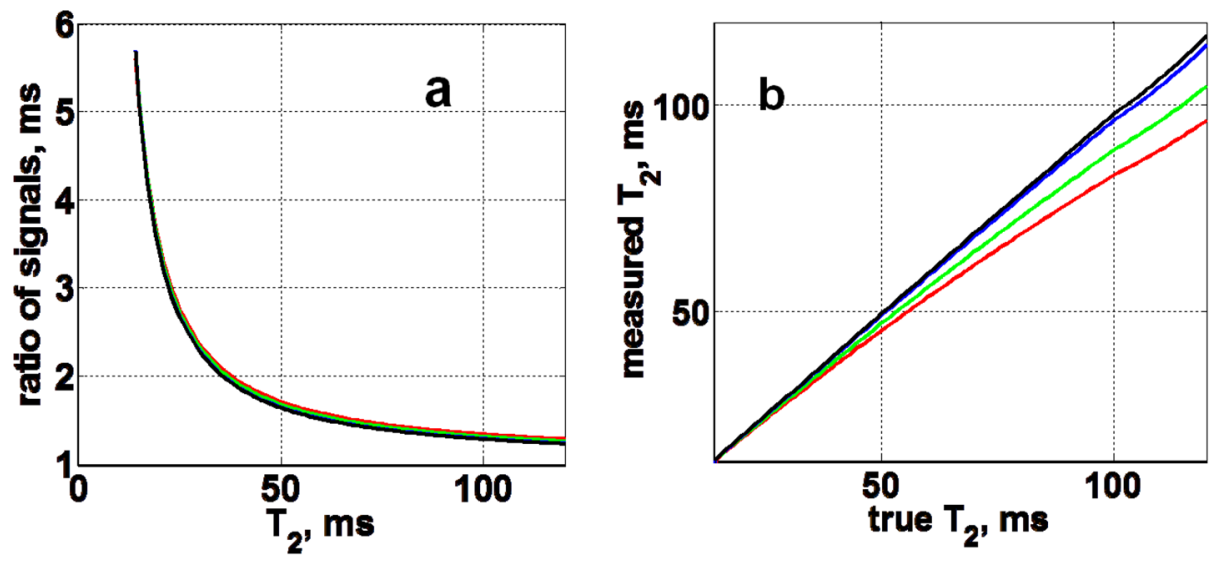

Fig 3.

(a) Computed ratio $R$ of two signals acquired with adiabatic pulses of length $\tau_{1}=5 \mathrm{~ms}$ with $\mathrm{g}=0.79$ for $f_{\max }=12 \mathrm{kHz}$ and $\tau_{2}=35 \mathrm{~ms}$ with $\mathrm{g}=0.81$ for $f_{\max }=15 \mathrm{kHz}$, as a function of $\mathrm{T}_{2}$ for

$\mathrm{T}_{1} \mathrm{~s}$ of $0.12-1 \mathrm{~s}$. The curve has the form $R\left(T_{2}\right)=\exp \left(\frac{-0.81 \times 35+0.79 \times 5}{T_{2}}\right)=\exp \left(\frac{-24.4}{T_{2}}\right)$. (b) Dual- $\tau \mathrm{T}_{2}$ determined from part (a) as a function of $\mathrm{T}_{1}$ (red, $\mathrm{T}_{1}=0.12 \mathrm{~s}$; green, $\mathrm{T} 1=0.2 \mathrm{~s}$; blue, $\mathrm{T}_{1}=0.6 \mathrm{~s}$; black, $\left.\mathrm{T} 1=1.0 \mathrm{~s}\right)$. The result is independent of $\mathrm{TR}$. 

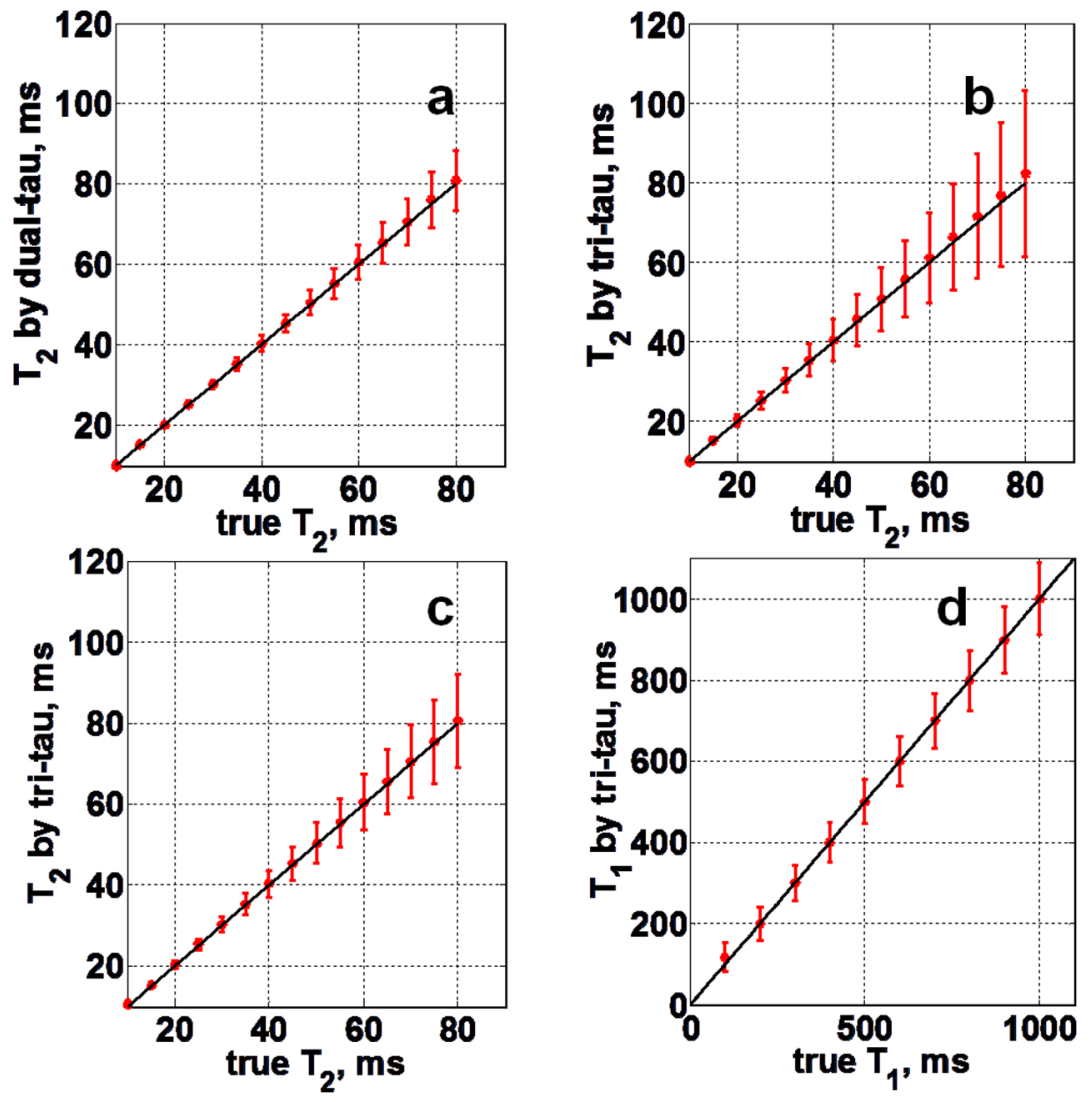

Fig 4.

Monte Carlo simulations of the error in (a) Dual- $\tau \mathrm{T}_{2}$ with $\tau_{1}=5 \mathrm{~ms}$ and $\tau_{2}=35 \mathrm{~ms}$; (b) Tri- $\tau$ $\mathrm{T}_{2}$ with $\tau_{3}=2 \tau_{2}=20 \mathrm{~ms}$; and (c) with $\tau_{3}=2 \tau_{2}=35 \mathrm{~ms}$; and (d) Tri- $\tau \mathrm{T}_{1}$ with $\tau_{3}=2 \tau_{2}=20 \mathrm{~ms}$, $\mathrm{T}_{2}=40 \mathrm{~ms}$, and $\mathrm{TR}=300 \mathrm{~ms}$. Points are means $\pm \mathrm{SD}$ for a $2 \%$ root-mean-square noise in each signal measurement $(\mathrm{SNR}=50)$ from 1000 runs with $\mathrm{T}_{1}=1 \mathrm{~s}$. The mean and $\mathrm{SD}$ of the $\mathrm{T}_{1}$ measured by a Tri- $\tau$ experiment with $\tau_{3}=2 \tau_{2}=35 \mathrm{~ms}$ differs from (d) by less than $1 \%$. 


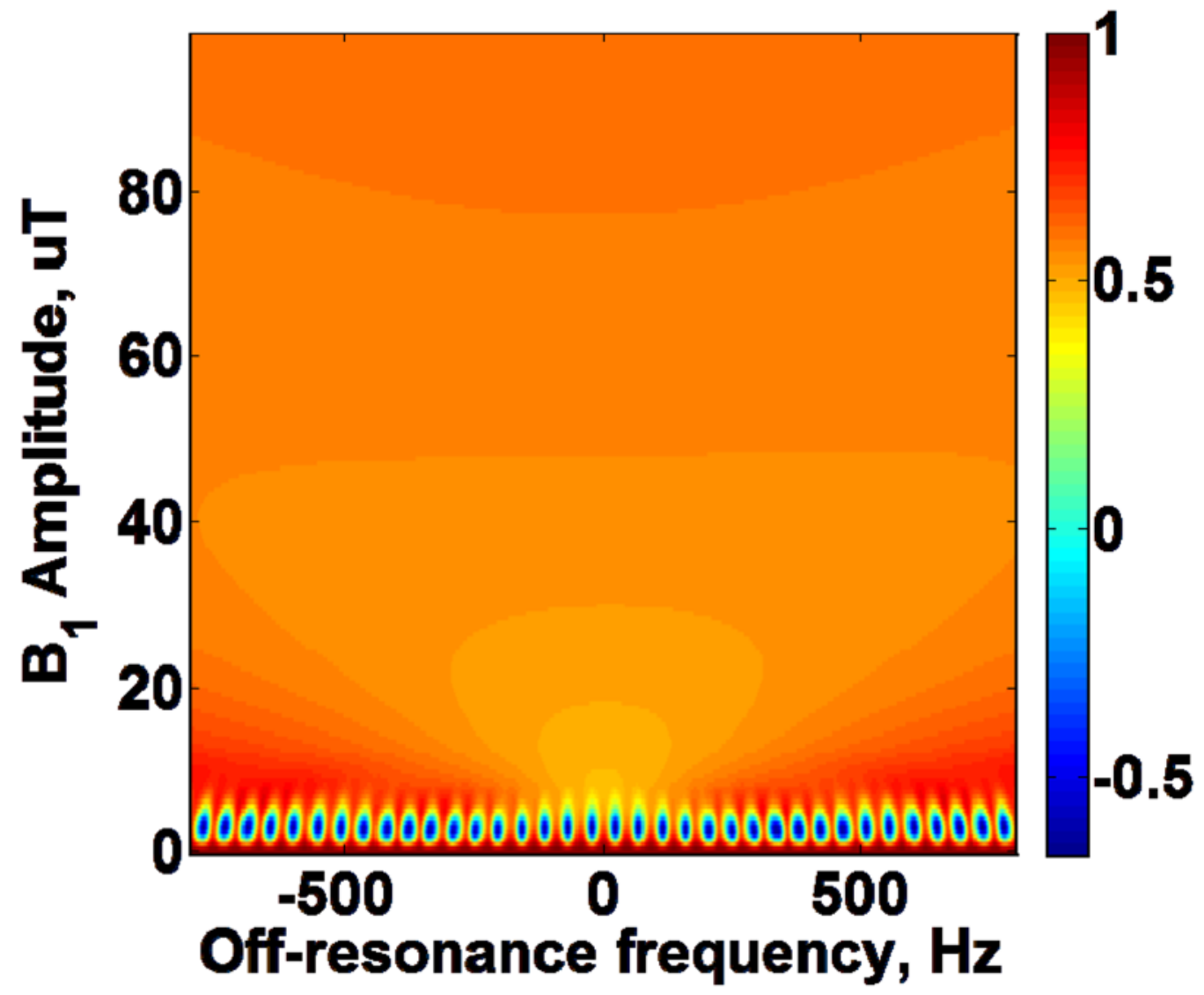

Fig 5.

Contour plot of $\mathrm{M}_{\mathrm{Z}} / \mathrm{M}_{\mathrm{equ}}$ (scale at right) as a function of off-resonance frequency using a $45 \mathrm{~ms} 0^{\circ}$ BIR- $4, T_{1}=1115 \mathrm{~ms}$, and $T_{2}=55 \mathrm{~ms}$, for comparison with the $45 \mathrm{~ms}_{2}$-prep sequence in Ref. [13]. The correct value of $\mathrm{M}_{\mathrm{z}} / \mathrm{M}_{\mathrm{equ}}=\exp \left(-0.81 * 45 / \mathrm{T}_{2}\right)=0.5$ (orange). 

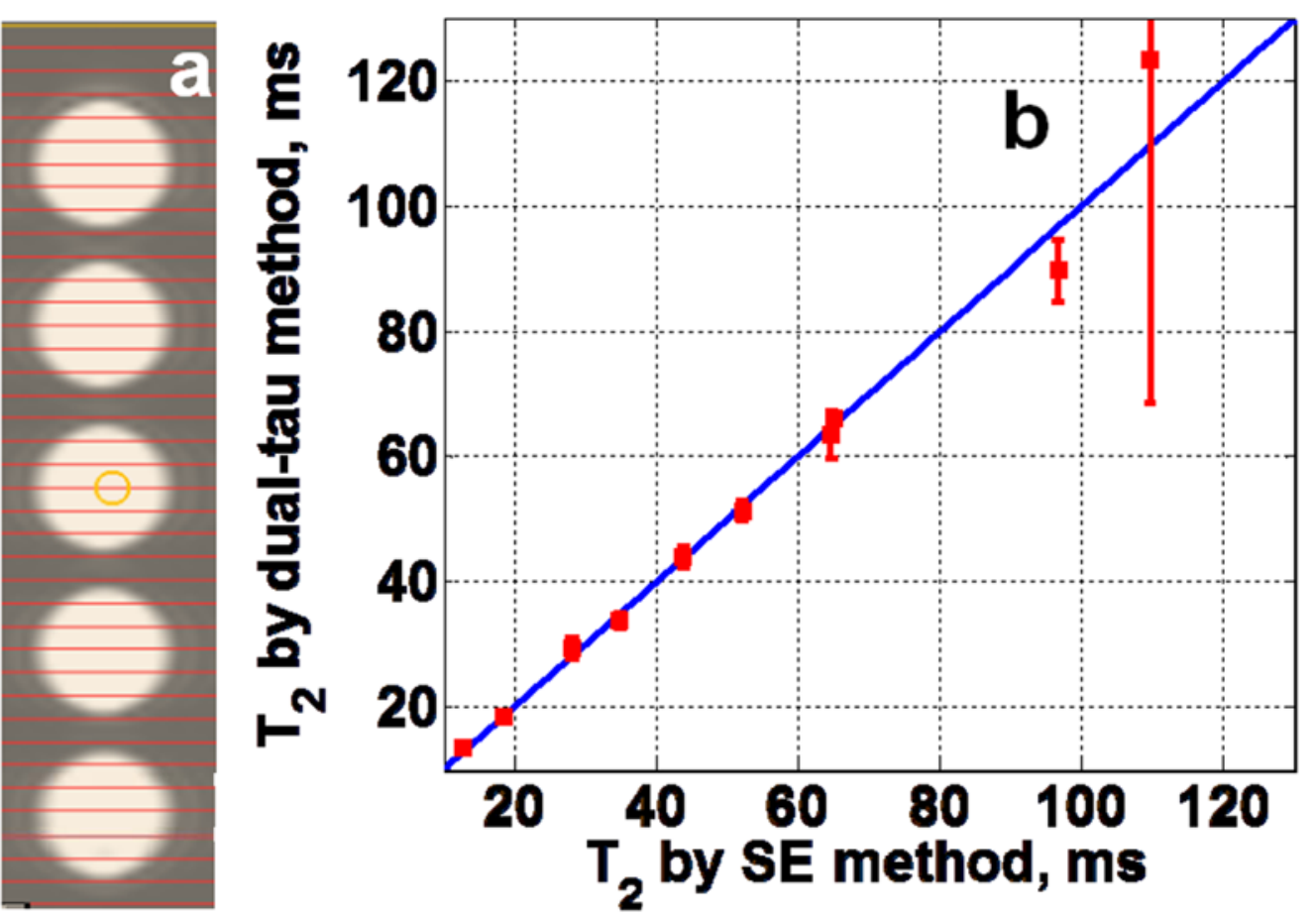

Fig 6.

(a) Transverse image of 5 of the phantoms annotated with $5 \mathrm{~mm}$ thick 1DCSI slices (annotated in red). (b) NMR measurements of $\mathrm{T}_{2}$ from the 10 phantoms using Dual- $\tau$ and SE methods. Filled points are the means of the middle three slices of each phantom. Error bars denote \pm SD. 

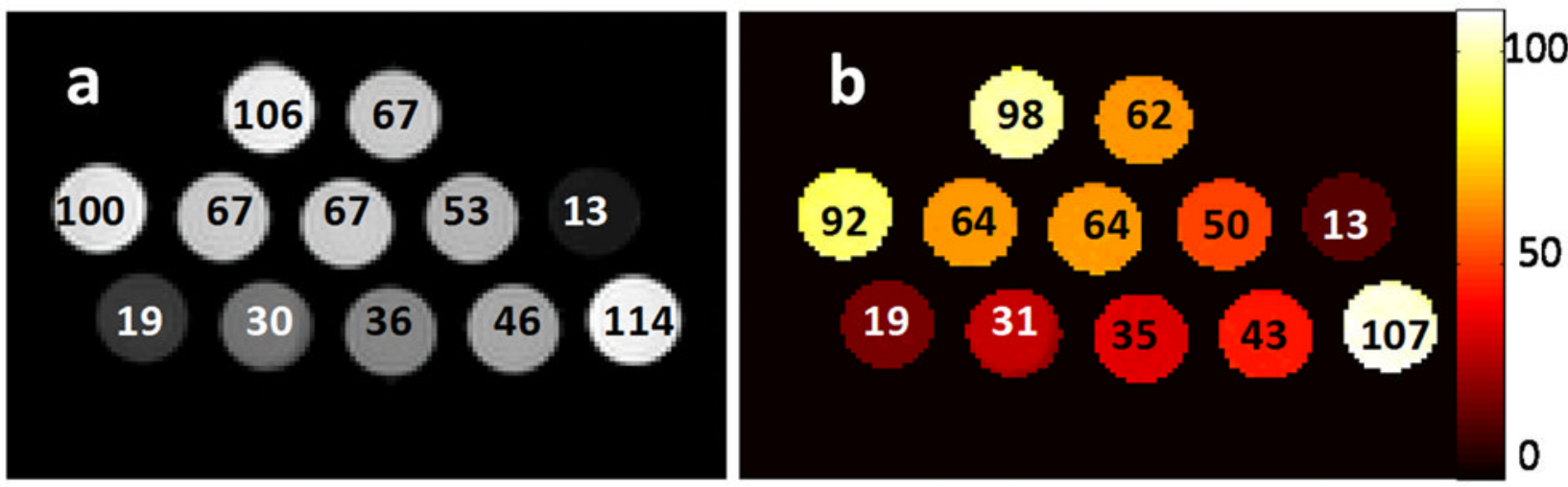

Fig 7.

(a) MRI of the phantom set, $\mathrm{T}_{2}$-weighted by a $0^{\circ} 35 \mathrm{~ms}$ BIR-4 pre-pulse. The images are annotated with the corresponding reference $S E T_{2}$. (b) Color-coded Dual- $\tau T_{2}$ image with the Dual- $\tau T_{2}$ values labeled for comparison with part (a).The scale depicts $T_{2}$ in $m s$. The $T_{2}$ map is calculated pixel by pixel, and both images masked at the same threshold $(=75 \%$ of the lowest signal in part a). 


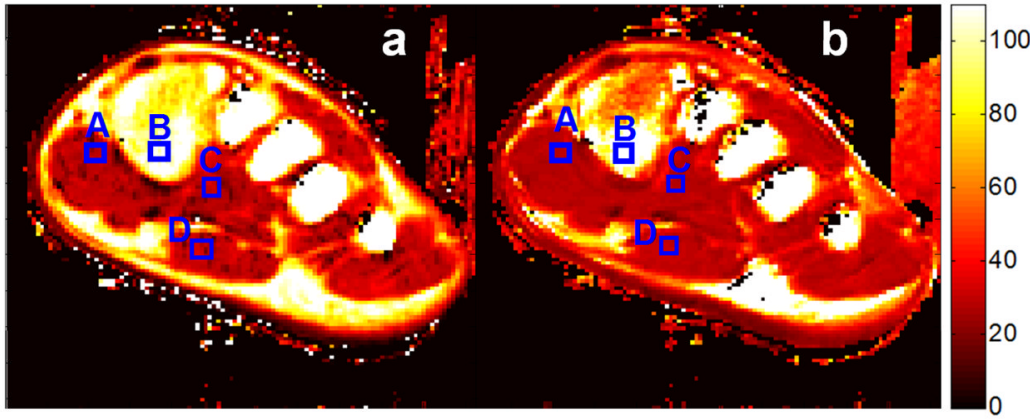

Fig 8.

Coronal $\mathrm{T}_{2}$ images of the human foot by the SE method (a) and the Dual- $\tau$ method (b). The scale depicts $\mathrm{T}_{2}$ in ms. Both images are calculated pixel-by-pixel and masked by an identical threshold ( $\mathrm{SNR}=4$ in the raw image). Mean $\mathrm{T}_{2}$ s from the annoted squares in (a) vs (b) are: A, $29.8 \pm 2.8 \mathrm{~ms}$ vs $29.4 \pm 1.2 \mathrm{~ms}$; B: $106 \pm 4.3 \mathrm{~ms}$ vs $135 \pm 13 \mathrm{~ms}$; C: $29.8 \pm 4.7 \mathrm{~ms}$ vs $28.2 \pm 1.6 \mathrm{~ms}$; D: $28.8 \pm 4.7 \mathrm{~ms}$ vs $30.6 \pm 1.3 \mathrm{~ms}$. 

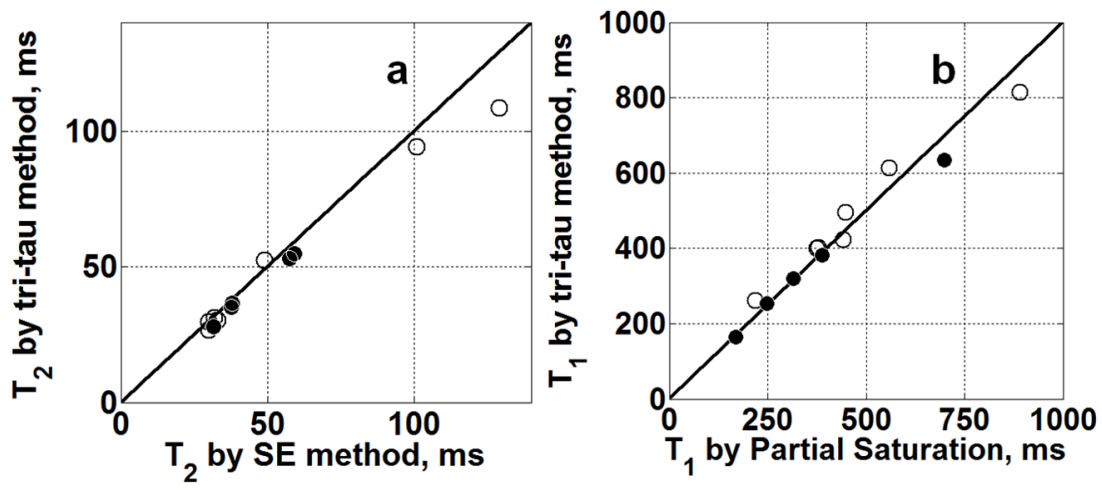

Fig 9.

$\mathrm{T}_{2}$ and $\mathrm{T}_{1}$ as measured by a $\tau_{3}=2 \tau_{2}=20 \mathrm{~ms}$ Tri- $\tau$ experiment from $12 \times 1.3 \mathrm{~cm} \mathrm{ID} \mathrm{(empty}$ points) and $4 \times 2.5 \mathrm{~cm}$ ID (filled points) phantoms, as compared with values measured using standard SE (a) and PS (b) methods (solid line =identity). 


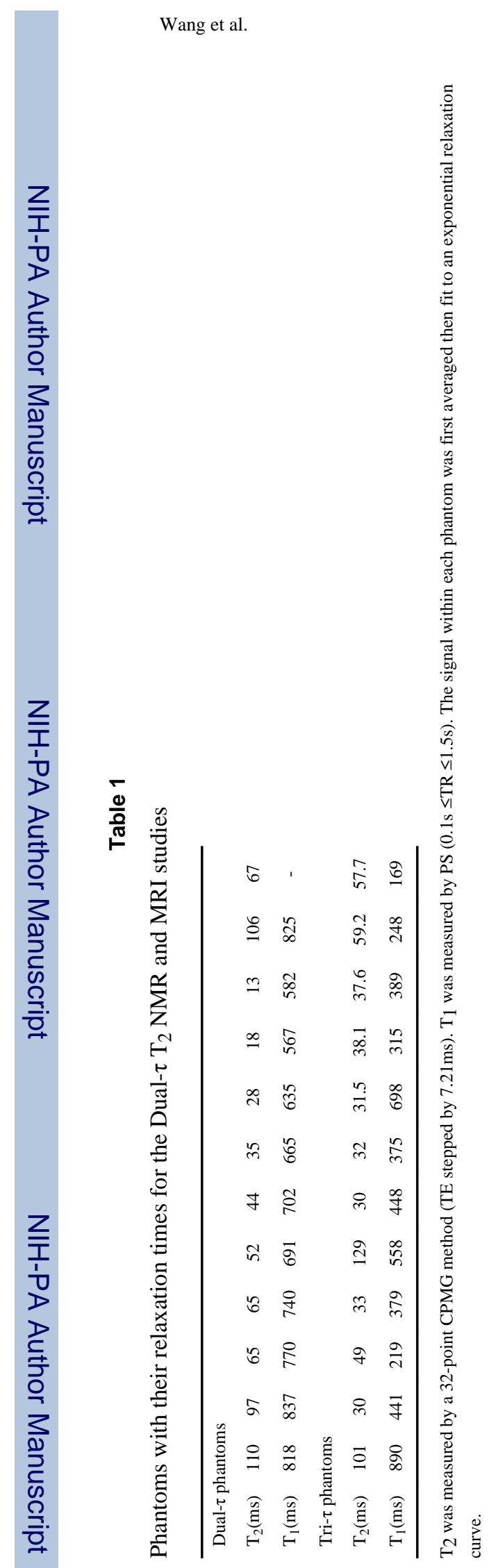

J Magn Reson. Author manuscript; available in PMC 2013 January 1. 